\title{
Prospecting prebiotics, innovative evaluation methods, and their health applications: a review
}

\author{
Ishu Khangwal ${ }^{1} \cdot$ Pratyoosh Shukla $^{1}$ (D)
}

Received: 27 December 2018 / Accepted: 12 April 2019 / Published online: 25 April 2019

๑) King Abdulaziz City for Science and Technology 2019

\begin{abstract}
Prebiotics are necessary natural and synthetic food ingredients that help in the growth and development of gut microflora. There is a complex relationship between gut dysbiosis and microbes, so alteration in both probiotics and prebiotics can reduce illness of gut, which further plays a decisive role in human health. The prebiotic efficiency can be validated using various in vitro and in vivo experiments, and this gives an important insight to this field. This review focuses on these aspects including the standardized assessment of prebiotics and its metabolic products for customary applications. This review has also summarized the mechanism of their beneficial actions such as immunomodulation, nutrient absorption, pathogen inhibition, etc., and its significance in human nutrition. In addition to this, some fascinating applications of prebiotics in health-related disorders have also discussed, with current challenges in this facet.
\end{abstract}

Keywords Prebiotics $\cdot$ Gut microbiota $\cdot$ Gastrointestinal tract $\cdot$ Oligosaccharides $\cdot$ Human health

\section{Introduction}

Prebiotics are food additives for the emerging manufacturers mainly in the food and dairy industries. These are nondigestible carbohydrate $(\mathrm{CHO})$ molecules, including disaccharides, oligosaccharides, polysaccharides, resistant starches, and sugar polyols. They also include other nondigestible substances which act as a food ingredient for gut microflora (Valdes et al. 2018). Prebiotics improves the health of the gut microflora by nourishing them in the gastrointestinal tract (GIT) (Zoumpopoulou et al. 2018). They enhance the growth and metabolism of gut microbes, by increasing the immune system, improving digestion, absorbing nutrients, and reducing the growth of pathogens which ultimately have positive effects on the human health (Gibson et al. 2017). The specific properties of prebiotics to survive in an acidic environment and resistant to the many enzymatic digestions in small intestine make them an incredible compound that can modulate gut microbiota. Furthermore, they can go to the large intestine where probiotics can ferment them into

Pratyoosh Shukla

pratyoosh.shukla@gmail.com

1 Enzyme Technology and Protein Bioinformatics Laboratory, Department of Microbiology, Maharshi Dayanand University, Rohtak, Haryana 124001, India short-chain fatty acids, vitamins, and other compounds (Fernando et al. 2018).

Prebiotics can be naturally occurring in various substances such as in banana asparagus, beans, mushrooms, and cereals; they can be synthesized by enzymatic digestion of polysaccharides and other complex materials (Geigerová et al. 2017). Some of the customary prebiotics products available in the market are inulin, fructooligosaccharide, galactooligosaccharide, and guar-gum (Madsen et al. 2017). Many dairy products such as fermented milk, cheese, and other milk beverages can be used for the transmission of lactosucrose and other prebiotics (Martín-Ortiz et al. 2017). While other xylan hydrolyzed prebiotic products such as xylooligosaccharides (XOS) and arabinoxylooligosaccharides (AXOS) are under developmental phase. Moreover, new potent sources of these can be identified for making cheap prebiotics products. Due to their high benefits, many pharmaceutical industries have a great interest in producing cost-efficient prebiotics. Synthetic approach is one of the most common ways for generating the present-day prebiotics which involves enzymatic digestion of residues to the high-quality prebiotic product. Beside this green chemistry, approach consists of the conversion of ordinary starch to resistant nature for developing new prebiotics (Vorholt et al. 2017). Prebiotic utilization varies from microbes to microbes, many of the gut microflora such as lactobacillus 
and bifidobacteria, etc. have different growth requirements to sustain in the GIT (Maleki et al. 2015). In general, gut microbiota takes prebiotic as nutrient sources for their metabolism and increase their population (Van Doan et al. 2018). This review will emphasize on various in vivo and in vitro strategies by which digestion and fermentation activity of prebiotics can be assessed. Evaluating prebiotics is essential as it can able to understand the digestion, fermentation, and products formed from them by the activity of gut microflora. Estimation of product formed by their action can be determined using different molecular strategies (Armenta et al. 2017). By their action sites, they have different mechanisms through which they perform their functions. They have different mechanisms for immunomodulation, pathogen inhibition, and compound activation (Holscher 2017). Healthy gut microflora helps in reducing the risk of many diseases such as obesity, diabetes, cardiovascular diseases, neurological disorders, cancer, etc., (Dahiya et al. 2017; Hill et al. 2017). Prebiotics are helpful in the proper functioning of the brain and controlling brain regulation (Alkasir et al. 2017). Specific evidence had been seen that they also have a crucial role in infant development (Noble et al. 2017). They are also helpful in curing bowel disease and specific oral health problems (Floch 2017). Moreover, new strategies such as encapsulation and immobilization can be used for developing new generation prebiotics (Díaz-Vergara et al. 2017). This review has also highlighted the current challenges in the production and evaluation strategies of prebiotics. Moreover, it can also give a possible idea of applying synthetic biology approach to this aspect.

\section{Evaluation of prebiotics}

Prebiotics are studied by different techniques based on three aspects, i.e., digestion, fermentation, and analysis (Bajury et al. 2017). For an appropriate prebiotic its non-digestibility should be tested, it should be resistant to gastric acidity, hydrolysis by mammalian enzymes, and gastrointestinal absorption. Each aspect of prebiotic is evaluated by different methodologies (Dey 2017). For such evaluations, the traditional media are used, comprising of yeast extract, sodium chloride, peptone water, calcium chloride hexahydrate, magnesium sulfate heptahydrate, Tween 80 , L-cysteine hydrochloride, vitamin K, haemin, resazurin, and bile salts (Doo et al. 2019). For the first step, i.e., digestion of prebiotics, it can be checked by in vitro and in vivo methodologies. The main aim is to mimic the human digestive tract at different stages of digestion. In the oral phase, conditions provided are $\mathrm{NaCl}$ and alpha-amylase for $5 \mathrm{~min}$ at $\mathrm{pH} 7$ (Garcia-Mazcorro et al. 2017). In vitro stomach process is complicated to mimic, at gastric phase, enzymes, pepsin, and gastric lipase are given for $2 \mathrm{~h}$ at $\mathrm{pH} 2.5$. After the gastric phase digestion of prebiotics can be checked at the last stage, i.e., the small intestine phases (Todorov et al. 2017). At small intestine phase enzymes such as chymotrypsin, trypsin, colipase, and pancreatic lipase are given for $2 \mathrm{~h}$ at $\mathrm{pH} 6$. In vivo digestion methodology is tested in the animal models, mainly rats and piglets, which are considered to be useful for these purposes (Chunchai et al. 2018). In these studies, digestion measurement of a substrate in the fecal and intubation into the gastrointestinal system of animal can be estimated. Moreover, in the distal ileum, undigested substrate can be envisaged while doing human clinical studies.

In the second step, i.e., fermentation of prebiotic compounds by intestinal microflora can be tested by in vitro and in vivo fermentation. In in vitro fermentation, methodology batch system, continues system, and the artificial gut system are used to check the prebiotic fermentations (ValdésVarela et al. 2017). In batch culture, $\mathrm{pH}$-based batch culture systems are used where the $\mathrm{pH}$ of the fermenter is maintained according to the appropriate level by applying gas production technique in these fermentations (Saman et al. 2017). Continuous culture fermentation is single staged or multi-staged based on the type of fermentation. TIM (The Netherlands Organization for Applied Scientific Research intestinal model) and SHIME ${ }^{\circledR}$ (The Simulator of the Human Intestinal Microbial Ecosystem) are two models in which the in vitro fermentation can be studied by the artificial gut system (Oliveira et al. 2017). In vivo fermentation methodology is done using animal models and human models. In animal models, fecal samples are collected after treating them with prebiotics. The human clinical study can be done directly by cumulating the breath air and indirectly by fecal collection (Oliveira et al. 2017).

The final step is an analysis of short-chain fatty acid and an increase in the gut microbes which can be done by various molecular strategies (Aryantini et al. 2017). Short-chain fatty acids (SCFs) are produced from the fermentation of carbohydrates by gut microbiota. In addition, they directly increase the microbial populations; so, it can be detected by gas chromatography (GC) and high-performance liquid chromatography (HPLC). Flame-ionization detection or mass spectrometric detection can be used in combination with gas chromatography for efficient detection. In HPLC, reversed phase column and ion exclusion column are commonly used for the screening. There is a diversified group of bacteria present in the gut that have a specific role in prebiotic fermentations; so, these can be detected by applying modern strategies. In early centuries, culture-based techniques were used to explore intestinal microflora from the human fecal. This technique is then moved to DNA-based culture-independent methods to detect a specific amount of DNA and RNA. The quantity of DNA is detected by real-time PCR or quantitative PCR (Rodriguez-Castaño et al. 2017). Pyrosequencing and 16s ribosomal RNA are 
common sequencing methods; in 16s RNA genes, a phylogenetic signal from different degrees of variability is used to differentiate bacterial populations (Yadav et al. 2018). In pyrosequencing, the emitted light from the synthesized DNA is captured and analyzed in a computational program such as primer express software and qbase+ (Foschi et al. 2017). Except for these DGGE (denaturing gradient gel electrophoresis) can be used to identify and characterize specific DNA pattern as a biomarker. DNA fingerprinting is done by TRFLP (terminal restriction fragment length polymorphism) which can be used for the comparison purposes, of gut microbiota diversity and variability (Reid 2008). Phylogenetic identification of gut microbiota can be made by DNA microarray technology and a fluorescence scanner which can detect probe-target duplex. The bacterial group can be identified using FISH (fluorescent in situ hybridization) where cells are fixed and hybridized on the glass slide and observed under a fluorescent microscope. Brief techniques for evaluating prebiotics are described in Fig. 1.

Moreover, different methods can be used for prebiotics analysis. The HILIC-ELSD method is used for the study of acetylated XOS and XOS. The technique is done on a zwitterionic HILIC column using ELSD detector. Structural information of X7, X8, and acetylated XOS can be determined by LC-ESI-MS (Xiao et al. 2018).

\section{Mechanism of beneficial action of prebiotics}

Prebiotics can affect host health by positively increasing the good intestinal microflora. These have an enormous impact on the intestinal microflora and its metabolic activity inducing health benefits. They cannot be digested by host enzymes; so, these are fermented by the gut bacteria the colon (Meurman and Stamatova 2018). Modulation in the

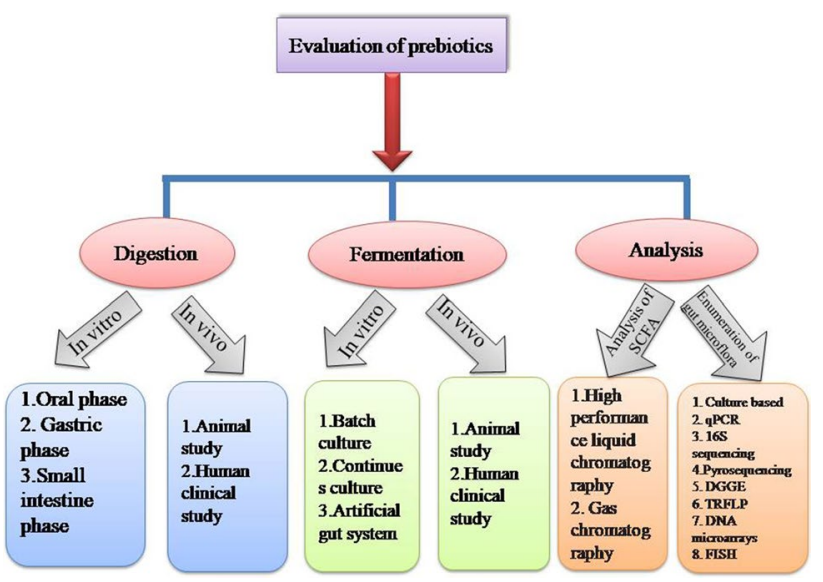

Fig. 1 In vivo and in vitro evaluation of prebiotics and their components using different techniques lipid metabolism can enhance the calcium absorbance which has a positive effect on immunological and bowel functions (Pekmez et al. 2018). Several trails on fishes have been made to investigate the action of prebiotics. Channastriata fingerlings were fed with selected prebiotics ( $\beta$-glucan, galactooligosaccharides, and maltooligosaccharides) and their growth performance, nutrient digestibility, the expression of immune regulatory gene, and retention was observed as compared to probiotics (Munir et al. 2016).

The actual mechanism of prebiotic utilization is not precise. Based on structure and composition, they can be utilized by specific bacteria which can use them as a carbon and energy source (Singh et al. 2017). Several proposed models have been seen which can show the prebiotic effect in the different parts of the body. Regulation of hepatic lipogenic enzyme can increase the production of short-chain fatty acid such as propionic acid, butyric acid as a result of fermentation (Vonk and Reckman 2017). Fermentation products increase the availability of various genes for transcriptional factors which enable the gut microflora to improve their population.

In spite, this certain prebiotic such as FOS can modulate mucin production (Alkasir et al. 2017). In GALTs (gutassociated lymphoid tissues) and peripheral blood, prebiotics can cause an increase in the lymphocytes as well as leukocyte count. GALTs then increase the IgA secretions which directly stimulate the phagocytic function of intrainflammatory macrophages. Prebiotics acts as nutrients for beneficial microorganisms which gives competition to the pathogens for epithelial adhesion (Holscher 2017). They produce antimicrobial substances by the mechanism of antagonism. SCFAs are the final product of carbohydrate metabolism, which may produce some pathogen inhibiting compounds and reduce intestinal $\mathrm{pH}$. Various studies showed inhibition of infectious pathogen by prebiotics (Chen and Gänzle 2017). Target mechanism of prebiotic action is explained in Fig. 2.

\section{Applications of prebiotics}

Prebiotics play a significant role in curing various human health-related issues, many studies have already been done showing the annihilation of diseases by continuous use of them (Yadav et al. 2017). Obesity is common worldwide; prebiotics can help in giving proper nutrition to the disturbed microflora, as in this disorder, human gut microflora is badly affected, it can increase the good bacterial growth affecting the health positively (Koenen et al. 2016). Not only obesity, there are many metabolic disorders in which intestinal microflora plays a major role such as in the proper functioning of the brain. Evidence has been seen that disruption in the gut microflora could directly affect the brain

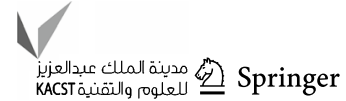




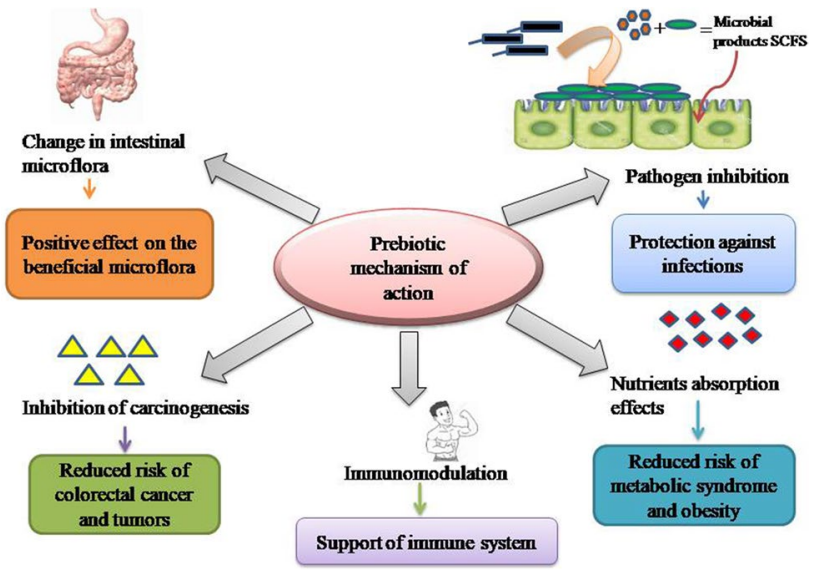

Fig. 2 Combinatory overview of mechanism of action of prebiotics showing metabolic efficiency and immunomodulation

functioning, the microbe-gut-brain axis has a vital role in the maintenance of both the brain and gut (Reid 2016). For instance, a combination of FOS and GOS was checked in C57BL/6J male mice, and it was seen that this combined prebiotic treatment reduces antidepressant and anxiolytic effects (Szklany et al. 2019). In the hippocampus, a hypothalamus-specific gene expression was modified by them. Chronic stress-induced elevations in corticosterone, proinflammatory cytokine levels, depression-like, and anxiety-like behavior can be reduced by FOS + GOS (Burokas et al. 2017; Serova et al. 2019). In infants also, prebiotics plays an essential role in maintaining the health of the baby. Prebiotics help in preventing various diseases such as diarrhea, infantile colic, necrotizing enterocolitis, celiac disease, allergies, obesity, neurologic disease, etc. Newborns have inoculated with oligosaccharide by breast milk fed to them. Corroboration studies of newborns have suggested that the presence of some fructooligosaccharide and galactooligosaccharide helps in the growth of Bifidobacteria and lactobacilli in their gut (Tomasello et al. 2016). Prebiotics helps in the management of irritable bowel syndrome (IBS) patients as prebiotics can help in maintaining the altered microflora which indirectly assists in reducing the symptoms. Prebiotics when was added to probiotics commonly known as symbiotics when administered in the diet improves the stool consistency, fermentation pattern, abdominal pain, bloating, and flatulence in patients with IBS (Ghoshal et al. 2016). Several studies have seen that probiotic can help in reducing dental caries and root caries arrest. Prebiotics and probiotics have significant effects in lowering cholesterol levels by improving the serum lipid profile studied using molecular docking approach (Rani et al. 2017). On the other hand, probiotics and prebiotics are also helpful in curing other cholesterol-related disorders such as the formation of abnormal erythrocytes by improving erythrocyte membrane fluidity, decreasing membrane rigidity, and altering membrane lipid profiles. They have health-enhancing effects, so pharmaceutical agencies can utilize them at an industrial scale. Industries have focused on producing safe, natural, and cost-effective prebiotics for improving health. Recently, a combination of probiotics with prebiotics has supplemented the individual use, which can improve the health of human (Mishra et al. 2018). Some of the probiotic and prebiotics strains which are used to cure various disorders are described in Table 1.

\section{Challenges to instigate prebiotics}

Microflora present in the gut is difficult to investigate; it plays a critical role in maintaining intestinal health including metabolism of different nutrients and prebiotics. There is no justification for the manipulated gut microflora, and it is hypothetical; the exact population and species of gut microbes is yet not known (Khaneghah and Fakhri 2019). Studying the combinatorial effects of prebiotics and probiotics for various diseases such as inflammatory bowel disease (IBD), necrotizing enterocolitis (NEC), encephalopathy, and ventilator-associated pneumonia (VAP) in intensive care units (ICU), etc. is not constant and contradictory (Yu-Ling et al. 2019). Moreover, in vivo and in vitro studies are difficult to mimic, for example an overriding species found in breast-fed infants, i.e., Bifidobacterium breve has antimicrobial activity against pathogens found in human gut having immuno-stimulation abilities also it is not cytotoxic and do not have infectious antibiotic resistance genes (Vandenplas and Savino 2019). Microbe varies from infected infants, especially with non-IgE mediated allergy, as compared to the healthy infants. In genetically predisposed individuals, the gluten exposure in diet can lead to chronic autoimmune celiac disease; these studies relate the role of gut microflora with clinical representations and pathogenesis of the disease. Combined probiotic treatment given to patients with this disease reduces the brutality of irritable bowel syndrome leading to the increase in the bifidobacteria populations. Extra care is required for the patients having such type of autoimmune disorders, malnutrition especially suffering from cancer (Bernal-Castro et al. 2019). Cautions for patients with special treatments need to be done; for example, probiotics intake via jejunostomy shows symptoms of ineffectual intestinal epithelial barrier associated with a wide range of antibiotics. In addition to this, the transmission mechanism of prebiotics is difficult; they need to be more focused and strategic for the enhancement of desired products (Khangwal and Shukla 2019a). Moreover, by applying systems, biology approach pathways for the metabolism of different target products can be known. It can also help in predicting the populations of gut microflora by knowing the byproducts 
Table 1 Combination of prebiotics and probiotics strains for treatment of various disorders

\begin{tabular}{|c|c|c|}
\hline Probiotic strain and prebiotic used & Disorder & References \\
\hline $\begin{array}{l}\text { Lactobacillus paracasei } \\
\text { Saccharomyces boulardii } \\
\text { Lactobacillus acidophilus } \\
\text { Galactooligosaccharide }\end{array}$ & Diarrhea & Hempel et al. (2012); Hasle (2018) \\
\hline $\begin{array}{l}\text { Streptococcusthermophilus, Lactobacillus, Bifidobac- } \\
\text { terium, etc. mixture of eight strains } \\
\text { Yogurt with Lactobacillusbulgaricus and Streptococ- } \\
\text { custhermophilus enriched with L. acidophilus and } \\
\text { Bifidobacterium lactis }\end{array}$ & Liver disease & $\begin{array}{l}\text { Shukla et al. (2011); Agrawal et al. (2012); } \\
\text { Zhao et al. (2015) }\end{array}$ \\
\hline $\begin{array}{l}\text { Bifidobacterium bifidum } \\
\text { SHORT-chain fructo-oligosaccharides } \\
\text { Galacto-oligosaccharides }\end{array}$ & IBS (Irritable bowel syndrome) & Guglielmetti et al. (2011); Ford et al. (2014) \\
\hline $\begin{array}{l}\text { Bifidobacterium lactis, Bifidobacteriumbifidum, Strep- } \\
\text { tococcus thermophiles, Lactobacillus acidophilus, } \\
\text { and Bifidobacterium longum } \\
\text { Lactulose } \\
\text { Fructo-oligosaccharide (FOS) and Lactobacilluspara- } \\
\text { casei, L. Rhamnosus, Lactobacillus acidophilus and } \\
\text { Bifidobacterium lactis }\end{array}$ & Functional constipation & Waitzberg et al. (2013); Yeun and Lee (2015) \\
\hline $\begin{array}{l}\text { Lactobacillus casei } \\
\text { Rifaximin and other fibers }\end{array}$ & $\begin{array}{l}\text { Uncomplicated symptomatic diverticular } \\
\text { disease }\end{array}$ & Tursi et al. (2013); Rezapour et al. (2018) \\
\hline
\end{tabular}

formed during the metabolisms of different substrates accordingly (Khangwal and Shukla 2019b).

\section{Conclusion and future perspectives}

Prebiotics has become accepted as a dietary food ingredient as they help in nourishing the gut microflora; they can be taken naturally from different foodstuff. These are short-chain oligosaccharide which is fermented by the gut microflora enhancing their growth. Use of them in the diet can improve human health and can prevent various health diseases, these can increase the absorption of multiple ions such as calcium and magnesium, and also these can help in controlling the blood glucose level and plasma lipids. They can be studied at different levels, such as digestion, fermentation, and analysis by in vivo and in vitro methodology. In addition to this, their action on various sites can directly affect the host body positively and targets in different locations having different roles in immunomodulation and suppressing carcinogens. New strategies for developing cost-effective and highly efficient prebiotics can be used. Moreover, the manufacturing cost of them can be reduced by extracting prebiotics from cheaper sources which are readily available. The interactions of microflora with them are still unknown, and so, 'metabolomics' can be applied to study these aspects in a detailed manner. Moreover, highly defined molecular approaches can be made for the gut microbes to understand the utilization and metabolism of these better.
Combining these two approaches can lead this story to the high peaks.

Acknowledgements The authors acknowledge Maharshi Dayanand University, Rohtak, India, for providing infrastructure facility. IK acknowledges the support as University Research Scholarship by M.D. University, Rohtak, India (Grant No. R\&S/R-15/19/1868). PS acknowledges the infrastructural support from Department of Science and Technology, New Delhi, Govt. of India, FIST grant (Grant No. 1196 SR/FST/LS-I/ 2017/4) and Department of Biotechnology, Government of India (Grant No. BT/PR27437/BCE/8/1433/2018). PS also acknowledges Department of Microbiology, Barkatullah University, Bhopal, India, for their infrastructural support for D.Sc. work.

\section{Compliance with ethical standards}

Conflict of interest All authors declare that they have no conflict of interest.

\section{References}

Agrawal A, Sharma BC, Sharma P, Sarin SK (2012) Secondary prophylaxis of hepatic encephalopathy in cirrhosis: an open-label, randomized controlled trial of lactulose, probiotics, and no therapy. Am J Gastroenterol 107:1043

Alkasir R, Li J, Li X et al (2017) Human gut microbiota: the links with dementia development. Protein Cell 8:90-102

Armenta S, Moreno-Mendieta S, Sánchez-Cuapio Z et al (2017) Advances in molecular engineering of carbohydrate-binding modules. Proteins Struct Funct Bioinforma 85:1602-1617

Aryantini NPD, Yamasaki E, Kurazono H et al (2017) In vitro safety assessments and antimicrobial activities of Lactobacillus rhamnosus strains isolated from a fermented mare's milk. Anim Sci J $88: 517-525$ 
Bajury DM, Nashri SM, King Jie Hung P, Sarbini SR (2017) Evaluation of potential prebiotics: a review. Food Rev Int 00:1-26

Bernal-Castro CA, Díaz-Moreno C, Gutiérrez-Cortés C (2019) Inclusion of prebiotics on the viability of a commercial Lactobacillus casei subsp. rhamnosus culture in a tropical fruit beverage. J Food Sci Technol 56:987-994

Burokas A, Arboleya S, Moloney RD et al (2017) Targeting the microbiota-gut-brain axis: prebiotics have anxiolytic and antidepressant-like effects and reverse the impact of chronic stress in mice. Biol Psychiatry 82:472-487

Chen XY, Gänzle MG (2017) Lactose and lactose-derived oligosaccharides: more than prebiotics? Int Dairy J 67:61-72

Chunchai T, Thunapong W, Yasom S et al (2018) Decreased microglial activation through gut-brain axis by prebiotics, probiotics, or synbiotics effectively restored cognitive function in obese-insulin resistant rats. J Neuroinflammation 15:11

Dahiya DK, Renuka Puniya M et al (2017) Gut microbiota modulation and its relationship with obesity using prebiotic fibers and probiotics: a review. Front Microbiol 8:563

Dey M (2017) Toward a personalized approach in prebiotics research. Nutrients 9:2015-2018

Díaz-Vergara L, Pereyra CM, Montenegro M et al (2017) Encapsulated whey-native yeast Kluyveromyces marxianus as a feed additive for animal production. Food Addit Contam Part A 34:750-759

Doo E-H, Schwab C, Chassard C, Lacroix C (2019) Cumulative effect of yeast extract and fructooligosaccharide supplementation on composition and metabolic activity of elderly colonic microbiota in vitro. J Funct Foods 52:43-53

Fernando WM, Flint SH, Ranaweera K et al (2018) The potential synergistic behaviour of inter-and intra-genus probiotic combinations in the pattern and rate of short chain fatty acids formation during fibre fermentation. Int J Food Sci Nutr 69:144-154

Floch MH (2017) The role of prebiotics and probiotics in gastrointestinal disease. Gastroenterol Clin North Am 47(1):179-191

Ford AC, Quigley EMM, Lacy BE et al (2014) Efficacy of prebiotics, probiotics, and synbiotics in irritable bowel syndrome and chronic idiopathic constipation: systematic review and meta-analysis. Am J Gastroenterol 109:1547

Foschi C, Laghi L, Parolin C et al (2017) Novel approaches for the taxonomic and metabolic characterization of lactobacilli: integration of 16S rRNA gene sequencing with MALDI-TOF MS and 1H-NMR. PLoS One 12:e0172483

Garcia-Mazcorro JF, Barcenas-Walls JR, Suchodolski JS, Steiner JM (2017) Molecular assessment of the fecal microbiota in healthy cats and dogs before and during supplementation with fructo-oligosaccharides (FOS) and inulin using high-throughput 454-pyrosequencing. PeerJ 5:e3184

Geigerová M, Bunešová V, Vlková E et al (2017) Selection of prebiotic oligosaccharides suitable for synbiotic use in calves. Anim Feed Sci Technol 229:73-78

Ghoshal U, Shukla R, Srivastava D, Ghoshal UC (2016) Irritable bowel syndrome, particularly the constipation-predominant form, involves an increase in Methanobrevibacter smithii, which is associated with higher methane production. Gut Liver 10:932

Gibson GR, Hutkins R, Sanders ME et al (2017) Expert consensus document: The international scientific association for probiotics and prebiotics (ISAPP) consensus statement on the definition and scope of prebiotics. Nat Rev Gastroenterol Hepatol 4(8):491

Guglielmetti S, Mora D, Gschwender M, Popp K (2011) Randomised clinical trial: Bifidobacterium bifidum MIMBb75 significantly alleviates irritable bowel syndrome and improves quality of life-a double-blind, placebo-controlled study. Aliment Pharmacol Ther 33:1123-1132

Hasle G (2018) Reply to comment "Do prebiotics reduce the risk of travelers 'diarrhea? Promising but more research needed." J Travel Med 25:tax086
Hempel S, Newberry SJ, Maher AR et al (2012) Probiotics for the prevention and treatment of antibiotic-associated diarrhea: a systematic review and meta-analysis. JAMA 307:1959-1969

Hill D, Sugrue I, Arendt E et al (2017) Recent advances in microbial fermentation for dairy and health. F1000Research 6:751

Holscher HD (2017) Dietary fiber and prebiotics and the gastrointestinal microbiota. Gut Microbes 8:172-184

Khaneghah AM, Fakhri Y (2019) Probiotics and prebiotics as functional foods: state of the art. Curr Nutr Food Sci 15:20-30

Khangwal I, Shukla P (2019a) Combinatory biotechnological intervention for gut microbiota. Appl Microbiol Biotechnol 103:3615-3625

Khangwal I, Shukla P (2019b) Potential prebiotics and their transmission mechanisms: recent approaches. J Food Drug Anal. https:// doi.org/10.1016/j.jfda.2019.02.003

Koenen ME, Rubio JMC, Mueller M, Venema K (2016) The effect of agave fructan products on the activity and composition of the microbiota determined in a dynamic in vitro model of the human proximal large intestine. J Funct Foods 22:201-210

Madsen LR, Stanley S, Swann P, Oswald J (2017) A survey of commercially available Isomaltooligosaccharide-based food ingredients. J Food Sci 82:401-408

Maleki D, Azizi A, Vaghef E, Balkani S, Homayouni A (2015) Methods of increasing probiotic survival in food and gastrointestinal conditions. Prensa Med Argent 101(4):1-9. https://doi. org/10.4172/lpma.1000154

Martín-Ortiz A, Barile D, Salcedo J et al (2017) Changes in caprine milk oligosaccharides at different lactation stages analyzed by high performance liquid chromatography coupled to mass spectrometry. J Agric Food Chem 65:3523-3531

Meurman JH, Stamatova IV (2018) Probiotics: evidence of oral health implications. Folia Med (Plovdiv) 60:21-29

Mishra SS, Behera PK, Kar B, Ray RC (2018) Advances in probiotics, prebiotics and nutraceuticals. In: Panda S, Shetty P (eds) Innovations in technologies for fermented food and beverage industries. Food microbiology and food safety. Springer, Cham, pp 121-141

Munir MB, Hashim R, Chai YH et al (2016) Dietary prebiotics and probiotics influence growth performance, nutrient digestibility and the expression of immune regulatory genes in snakehead (Channa striata) fingerlings. Aquaculture 460:59-68

Noble EE, Hsu TM, Kanoski SE (2017) Gut to brain dysbiosis: mechanisms linking western diet consumption, the microbiome, and cognitive impairment. Front Behav Neurosci 11:9

Oliveira D, Vidal L, Ares G et al (2017) Sensory, microbiological and physicochemical screening of probiotic cultures for the development of non-fermented probiotic milk. LWT Food Sci Technol 79:234-241

Pekmez CT, Dragsted LO, Brahe LK (2018) Gut microbiota alterations and dietary modulation in childhood malnutrition - the role of short chain fatty acids. Clin Nutr 32(2):615-630

Rani RP, Anandharaj M, Ravindran AD (2017) Characterization of bile salt hydrolase from Lactobacillus gasseri FR4 and demonstration of its substrate specificity and inhibitory mechanism using molecular docking analysis. Front Microbiol 8:1004

Reid G (2008) Probiotics and prebiotics-progress and challenges. Int Dairy J 18:969-975

Reid G (2016) Probiotics: definition, scope and mechanisms of action. Best Pract Res Clin Gastroenterol 30:17-25

Rezapour M, Ali S, Stollman N (2018) Diverticular disease: an update on pathogenesis and management. Gut Liver 12:125

Rodriguez-Castaño GP, Caro-Quintero A, Reyes A, Lizcano F (2017) Advances in gut microbiome research, opening new strategies to cope with a western lifestyle. Front Genet 7:224

Saman P, Tuohy KM, Vázquez JA (2017) In vitro evaluation of prebiotic properties derived from rice bran obtained by debranning technology. Int J Food Sci Nutr 68:421-428 
Serova LI, Nwokafor C, Van Bockstaele EJ, Reyes BA, Lin X, Sabban EL (2019) Single prolonged stress PTSD model triggers progressive severity of anxiety, altered gene expression in locus coeruleus and hypothalamus and effected sensitivity to NPY. Eur Neuropsychopharmacol 29:482-492

Shukla S, Shukla A, Mehboob S, Guha S (2011) Meta-analysis: the effects of gut flora modulation using prebiotics, probiotics and synbiotics on minimal hepatic encephalopathy. Aliment Pharmacol Ther 33:662-671

Singh RK, Chang H-W, Yan D (2017) Influence of diet on the gut microbiome and implications for human health. J Transl Med 15:73

Szklany K, Wopereis H, de Waard C et al (2019) Supplementation of dietary non-digestible oligosaccharides from birth onwards improve social and reduce anxiety-like behaviour in male BALB/c mice. Nutr Neurosci. https://doi.org/10.1080/10284 15X.2019.1576362

Todorov SD, Holzapfel W, Nero LA (2017) In Vitro evaluation of beneficial properties of bacteriocinogenic Lactobacillus plantarum ST8Sh. Probiotics Antimicrob Proteins 9:194-203

Tomasello G, Mazzola M, Leone A (2016) Nutrition, oxidative stress and intestinal dysbiosis: influence of diet on gut microbiota in inflammatory bowel diseases. Biomed Pap Fac Med Palacký Univ Olomouc Czech Repub 4:461-466

Tursi A, Brandimarte G, Elisei W (2013) Randomised clinical trial: mesalazine and/or probiotics in maintaining remission of symptomatic uncomplicated diverticular disease - a double-blind, randomised, placebo-controlled study. Aliment Pharmacol Ther 38:741-751

Valdes AM, Walter J, Segal E, Spector TD (2018) Role of the gut microbiota in nutrition and health. BMJ 361:k2179

Valdés-Varela L, Ruas-Madiedo P, Gueimonde M (2017) In vitro fermentation of different fructo-oligosaccharides by bifidobacterium strains for the selection of synbiotic combinations. Int J Food Microbiol 242:19-23

Van Doan H, Hoseinifar SH, Faggio C (2018) Effects of corncob derived xylooligosaccharide on innate immune response, disease resistance, and growth performance in Nile tilapia (Oreochromis niloticus) fingerlings. Aquaculture 495:786-793

Vandenplas Y, Savino F (2019) Probiotics and prebiotics in pediatrics: what Is new? Nutrients 11(2)-431

Vonk RJ, Reckman G (2017) Progress in the biology and analysis of short chain fatty acids. J Physiol 595:419-420

Vorholt JA, Vogel C, Carlström CI, Müller DB (2017) Establishing causality: opportunities of synthetic communities for plant microbiome research. Cell Host Microbe 22:142-155

Waitzberg DL, Logullo LC, Bittencourt AF (2013) Effect of synbiotic in constipated adult women - a randomized, double-blind, placebo-controlled study of clinical response. Clin Nutr 32:27-33

Xiao X, Wen J-Y, Wang Y-Y (2018) NMR and ESI-MS spectrometry characterization of autohydrolysis xylo-oligosaccharides separated by gel permeation chromatography. Carbohydr Polym 195:303-310

Yadav R, Singh PK, Puniya AK, Shukla P (2017) Catalytic interactions and molecular docking of bile salt hydrolase (BSH) from 1. plantarum RYPR1 and its prebiotic utilization. Front Microbiol 7:1-7

Yadav R, Kumar V, Baweja M, Shukla P (2018) Gene editing and genetic engineering approaches for advanced probiotics: a review. Crit Rev Food Sci Nutr 58:1735-1746

Yeun Y, Lee J (2015) Effect of a double-coated probiotic formulation on functional constipation in the elderly: a randomized, double blind, controlled study. Arch Pharm Res 38:1345-1350

Yu-Ling T, Lin T-L, Chih-Jung C (2019) Probiotics, prebiotics and amelioration of diseases. J Biomed Sci 26:1-3

Zhao H, Zhao C, Dong Y (2015) Inhibition of miR122a by Lactobacillus rhamnosus GG culture supernatant increases intestinal occludin expression and protects mice from alcoholic liver disease. Toxicol Lett 234:194-200

Zoumpopoulou G, Kazou M, Alexandraki V (2018) Probiotics and prebiotics: an overview on recent trends. In: Probiotics and prebiotics in animal health and food safety. Springer, New York, pp $1-34$ 